OPEN ACCESS

Edited by:

Patricio Ramos,

Universidad Católica de Maule, Chile

Reviewed by:

Cristian Atala,

Pontificia Universidad Católica

de Valparaíso, Chile

Aparna Banerjee,

Catholic University of the Maule, Chile

*Correspondence:

Marcia González-Teuber

mfgonzalez@ucsc.cl

Luisa Bascuñán-Godoy

lubascun@udec.cl

Specialty section: This article was submitted to

Coevolution,

a section of the journa

Frontiers in Ecology and Evolution

Received: 30 September 2021

Accepted: 06 December 2021

Published: 07 January 2022

Citation:

González-Teuber M,

Contreras RA, Zúñiga GE, Barrera $D$

and Bascuñán-Godoy L (2022)

Synergistic Association With Root

Endophytic Fungi Improves

Morpho-Physiological and

Biochemical Responses of

Chenopodium quinoa to Salt Stress.

Front. Ecol. Evol. 9:787318.

doi: 10.3389/fevo.2021.787318

\section{Synergistic Association With Root Endophytic Fungi Improves Morpho-Physiological and Biochemical Responses of Chenopodium quinoa to Salt Stress}

\author{
Marcia González-Teuber ${ }^{*}$, Rodrigo A. Contreras², Gustavo E. Zúñiga², Diego Barrera ${ }^{3}$ \\ and Luisa Bascuñán-Godoy ${ }^{3 *}$
}

${ }^{1}$ Laboratorio de Ecología Química, Departamento de Química Ambiental, Facultad de Ciencias, Universidad Católica de la Santísima Concepción, Concepción, Chile, ${ }^{2}$ Laboratorio de Fisiología y Biotecnología Vegetal, Departamento de Biología, Facultad de Química y Biología, Universidad de Santiago de Chile, Santiago, Chile, ${ }^{3}$ Laboratorio de Fisiología Vegetal, Departamento de Botánica, Facultad de Ciencias Naturales y Oceanográficas, Universidad de Concepción, Concepción, Chile

Symbiotic associations with microbes can contribute to mitigating abiotic environmental stress in plants. In this study, we investigated individual and interactive effects of two root endophytic fungal species on physiological and biochemical mechanisms of the crop Chenopodium quinoa in response to salinity. Fungal endophytes (FE) Talaromyces minioluteus and Penicillium murcianum, isolated from quinoa plants that occur naturally in the Atacama Desert, were used for endophyte inoculation. A greenhouse experiment was developed using four plant groups: (1) plants inoculated with T. minioluteus $(\mathrm{E} 1+)$, (2) plants inoculated with P. murcianum $(\mathrm{E} 2+)$, (3) plants inoculated with both fungal species $(\mathrm{E} 1 \mathrm{E} 2+)$, and (4) non-inoculated plants (E-). Plants from each group were then assigned to either salt $(300 \mathrm{mM})$ or control (no salt) treatments. Differences in morphological traits, photosynthesis, stomatal conductance, transpiration, superoxide dismutase (SOD), ascorbate peroxidase (APX), peroxidase, $(\mathrm{POD})$, phenylalanine ammonia-lyase $(\mathrm{PAL})$, phenolic content, and lipid peroxidation between plant groups under each treatment were examined. We found that both endophyte species significantly improved morphological and physiological traits, including plant height, number of shoots, photosynthesis, stomatal conductance, and transpiration, in C. quinoa in response to salt, but optimal physiological responses were observed in E1E2+ plants. Under saline conditions, endophyte inoculation improved SOD, APX, and POD activity by over $50 \%$, and phenolic content by approximately $30 \%$, with optimal enzymatic responses again observed in E1E2+ plants. Lipid peroxidation was significantly lower in inoculated plants than in non-inoculated plants. Results demonstrate that both endophyte species enhanced the ability of $C$. quinoa to cope with salt stress by improving antioxidative enzyme and non-enzyme systems. In general, 
both FE species interacting in tandem yielded better morphological, physiological, and biochemical responses to salinity in quinoa than inoculation by a single species in isolation. Our study highlights the importance of stress-adapted FE as a biological agent for mitigating abiotic stress in crop plants.

Keywords: symbiotic fungi, salinity, plant tolerance, synergic effects, physiological performance, lipid peroxidation, antioxidant enzymes, phenols

\section{INTRODUCTION}

Abiotic stresses linked to climate change are a major factor restricting plant growth and distribution (Bartels and Sunkar, 2005). Increases in soil salinity can result in considerable declines in plant performance by producing an ion imbalance and hyperosmotic stress (Zhu, 2001), which limits plant water uptake. Similarly, high soil salinity has deleterious effects on several physiological and biochemical processes, including declines in photosynthesis and stomatal conductance, and can lead to an increase in oxidative damage through the formation of reactive oxygen species (ROS) (Sharma et al., 2005; Bose et al., 2014; Hnilickova et al., 2021). An excess of ROS may cause severe damage to proteins, lipids, membranes, and DNA, leading to an increase in lipid peroxidation (Foyer and Noctor, 2003; Meloni et al., 2003; Apel and Hirt, 2004; Ozgur et al., 2015). In response to ROS, plants may activate an array of enzymatic and non-enzymatic antioxidant mechanisms, including superoxide dismutase (SOD), peroxidase (POD), catalase (CAT), ascorbate peroxidase (APX), phenylalanine ammonia-lyase (PAL), and secondary metabolites with antioxidant properties, such as phenolic compounds (Meloni et al., 2003; Ozgur et al., 2013; Al Hassan et al., 2017).

Associations with symbiotic endophytic fungi are recognized as a key component of plant responses to abiotic stress (Rodriguez et al., 2008). Horizontally transmitted fungal endophytes (FE) are ubiquitous in plants and colonize a variety of plant tissues (Rodriguez et al., 2009). Whereas most FE are defined as commensalistic, with no or yet unknown functions in plants, there are several reports of fungal symbionts improving host resilience to a range of abiotic stresses, including drought, heat, and salinity (Baltruschat et al., 2008; Singh et al., 2011; Bagheri et al., 2013; AcuñaRodríguez et al., 2019; Gupta et al., 2021; Moghaddam et al., 2021). Interestingly, stress tolerance conferred by some FE taxa seems to involve habitat-specific fungal adaptations, i.e., fungal species isolated from plants occurring in areas characterized by high levels of environmental stress are particularly effective at enhancing host stress tolerance (Rodriguez et al., 2008; Giauque et al., 2018). For example, wheat seedlings inoculated with saline-adapted Azospirillum fungal strains have been found to have higher levels of photosynthetic pigments and proline accumulation than seedlings inoculated with non-salineadapted Azospirillum strains (Azad and Kaminskyj, 2016). Consequently, several studies have reported that stress-adapted FE are able to mitigate negative effects of salinity by improving a range of physiological and biochemical plant responses, including photosynthesis, transpiration rate, antioxidant enzyme activity, and concentrations of osmoprotectant molecules, such as proline and soluble sugars (Rodriguez et al., 2008; Zarea et al., 2012; Azad and Kaminskyj, 2016; Li et al., 2017; Molina-Montenegro et al., 2020; Moghaddam et al., 2021). In this sense, stress-adapted FE may potentially be used as biological agents to assist in mitigating abiotic stress in plants.

There is some evidence to suggest that the effects of FE may be synergistic or additive in nature, where the presence of several mutualists results in increased host benefits (Gazis and Chaverri, 2015; González-Teuber, 2016; Bilal et al., 2020). While the benefits of FE in terms of alleviating abiotic stresses have usually been demonstrated using a single FE species, synergistic effects of several FE taxa in combination have rarely been investigated. Results from a recent study, however, suggest that cooperation among endophytes leads to enhanced plant responses to combined abiotic stresses (Bilal et al., 2020). It was shown that soybean plants co-inoculated with two FE species displayed improved growth, photosynthesis, and antioxidant mechanisms in response to heavy metals, high temperature, and drought stress compared to single-inoculated and non-inoculated plants (Bilal et al., 2020). These results highlight the importance of multiple symbiotic agents interacting to ameliorate plant stress. For agricultural food crops in particular, combining symbiotic microorganisms may be useful in terms of developing and improving management strategies.

Chenopodium quinoa is a pseudo-cereal crop of the Amaranthaceae family, native to the Andean region of South America. Quinoa is an important crop due to its high protein content and resilience to a range of stressful conditions (Bascuñán-Godoy et al., 2016; Lutz and Bascuñán-Godoy, 2017). In Chile, it occurs naturally in the Atacama Desert (Rodriguez et al., 2008; Fuentes and Bhargava, 2011), where extreme climatic conditions, including heat, drought, and soil salinity (Houston and Hartley, 2003) are key constraints to plant growth and distribution. It has been suggested that colonization by microbial symbionts potentially ameliorates adverse effects of abiotic stresses in quinoa plants (González-Teuber et al., 2018). Earlier research shows that Talaromyces minioluteus (former Penicillium minioluteum) and Penicillium murcianum are the most abundant FE colonizing healthy quinoa root tissues growing near the Salar de Atacama (González-Teuber et al., 2017), with $P$. minioluteum observed to improve host tolerance to water stress (González-Teuber et al., 2018). In this study, quinoa plants were colonized with T. minioluteus and P. murcianum and exposed to a salinity treatment. Our goal was to assess whether individual and interactive effects of both FE enhance the ability of C. quinoa to tolerate salinity, including morphological (plant height and number of shoots), physiological (photosynthesis, stomatal 
conductance, and transpiration) and biochemical mechanisms (antioxidant enzymes, phenolic content, and lipid peroxidation). Based on the natural occurrence of both endophyte species in saline environments, we predicted that FE would increase tolerance to salinity in C. quinoa. Additionally, we predicted that co-inoculated plants would exhibit greater salt tolerance mechanisms than single and non-inoculated plants.

\section{MATERIALS AND METHODS}

\section{Study Site and Sample Collection}

Chenopodium quinoa Willd. (Amaranthaceae) is a gynomonoecious annual plant with an erect stem. It bears alternate leaves that are variously colored due to the presence of betacyanins. The inflorescence is a panicle of $15-70 \mathrm{~cm}$ in length, rising from the top of the plant and from the axils of lower leaves (Bhargava et al., 2006). Seeds were collected from plants growing near the village of Socaire $\left(23^{\circ} 36^{\prime} 00^{\prime \prime} \mathrm{S}\right.$ and $\left.67^{\circ} 50^{\prime} 60^{\prime \prime} \mathrm{W}\right)$, situated $3.500 \mathrm{~m}$ above sea level, $50 \mathrm{~km}$ East of the Salar de Atacama. The climate in the Atacama Desert is characterized as extremely arid (Noy-Meyr, 1973). Daily average temperatures range from 7.1 to $24.5^{\circ} \mathrm{C}$, with a mean annual precipitation of $18 \mathrm{~mm}$ (García et al., 2007). In a recent study (González-Teuber et al., 2017) characterizing the fungal endophyte community associated with roots of healthy quinoa plants growing in the Atacama Desert (Supplementary Figure 1), Talaromyces and Penicillium were identified as the dominant genera, with T. minioluteus (former Penicillium minioluteum) and $P$. murcianum constituting the two most abundant FE (González-Teuber et al., 2017). Inoculation experiments developed here were conducted using T. minioluteus (NCBI ID: OL634957) and P. murcianum strains (NCBI ID: OL634958). Pure cultures of each were obtained from our laboratory collection. Cultures were grown for 14 days at $28^{\circ} \mathrm{C}$ in $16 \mathrm{~mm}$ petri dishes with yeast malt agar (YMA). YMA (Atlas and Parks, 1993) was used as it has been found to be a suitable medium for spore production in both endophyte species (González-Teuber, personal observations). Spores were then collected by repetitively flooding the agar plates with distilled sterile water and rubbing the surface with a sterile spatula. Samples were transferred to sterile bottles for storage. A Neubauer chamber cell counter (HBG Company, Germany) was used to adjust the spore concentration to $1 \times 10^{7}$ spores $\mathrm{mL}^{-1}$. This concentration was later used to inoculate plant roots.

\section{Plant Material and Salinity Experiment}

Seeds were surface sterilized by immersion in $0.5 \%$ sodium hypochlorite for $3 \mathrm{~min}$ (Sauer and Burroughs, 1986). They were subsequently rinsed with sterile distilled water and dried using sterile paper towel. The effectiveness of the surface sterilization method was confirmed by the absence of any microbial growth on PDA (potato dextrose agar) plates (PhytoTechnology Laboratories), evaluated by plating water used for the final rinse. Germinated seeds were transplanted to $0.2 \mathrm{~L}$ plastic pots filled with a mixture of sterilized soil and sand (1:1) and grown in a growth chamber under controlled conditions $\left(400 \mu \mathrm{mol} \mathrm{m} \mathrm{m}^{-2}\right.$ $\mathrm{s}^{-1}$ PAR (photosynthetically active radiation), with a minimum temperature of $17^{\circ} \mathrm{C}$ and a maximum of $23^{\circ} \mathrm{C}$. Seedlings were moderately irrigated every second day to ensure establishment. Two weeks after transplanting, plants were separated into four groups: (1) non-inoculated plants (E-), (2) plants inoculated with T. minioluteus $(\mathrm{E} 1+)$, (3) plants inoculated with $P$. murcianum $(\mathrm{E} 2+)$, and (4) plants inoculated with both fungal species $(\mathrm{E} 1 \mathrm{E} 2+)$. Non-inoculated plants $(N=10$ plants) were irrigated with sterile water free of endophyte spores. Single endophyte inoculation was achieved by watering plants either with a spore solution of T. minioluteus or $P$. murcianum ( $N=10$ plants in each case; concentration adjusted to $1 \times 10^{7}$ spores $\left.\mathrm{mL}^{-1}\right)$. For coinoculation, plants were irrigated with a mixture containing both endophyte species (1:1) $(N=10$ plants; concentration adjusted to $1 \times 10^{7}$ spores $\left.\mathrm{mL}^{-1}\right)$. Non-inoculated plants $(N=10$ plants $)$ were irrigated with sterile water free of endophyte spores. After 3 weeks of FE inoculation, just prior to the salinity treatment, three E- and E+ plants were sacrificed in order to verify presence and absence of T. minioluteus and P. murcianum. This was confirmed by collecting root material and testing for the presence of both fungal species through isolation of the endophytes on culture media (Arnold et al., 2000). E- plants were free of endophytic fungi in their roots. Plants from each endophyte group were then assigned to either salt $(300 \mathrm{mM}$ of $\mathrm{NaCl}$ ) or control (no salt) treatments, resulting in a total of eight plant groups. A salt concentration of $300 \mathrm{mM}$ was applied, since this concentration affects physiological performance in C. quinoa (Ruiz et al., 2016, 2017). The experiment was run as a full-factorial completely randomized design. Morphological and physiological traits were measured after a period of 3 weeks, including plant height, number of shoots, photosynthesis, stomatal conductance, and transpiration. Additionally, leaf material from all plants was collected and stored at $-80^{\circ} \mathrm{C}$ to perform measurements of antioxidative enzyme activity (SOD, APX, POD, and PAL), total phenolic content, and lipid peroxidation.

\section{Morphological Measurements}

For each plant group ( $N=5$ plants per treatment), plant height $(\mathrm{cm})$ was measured from the ground level to the tip of the main stem. The total number of shoots was recorded by counting all branches emerging from the main stem at different node positions, including the basal branches. Although plant biomass was not measured at the end of the experiment, a previous study in C. quinoa showed that the number of shoots is positively correlated with final biomass (Shah et al., 2020). Moreover, both traits, plant height and number of shoots, have been demonstrated to be susceptible to salinity in halophyte plants, including C. quinoa (Vasquez et al., 2006; Hussain et al., 2020; Jaramillo Roman et al., 2020).

\section{Gas Exchange Measurements}

For each plant group, gas-exchange measurements were conducted in fully expanded leaves (third leaf from the top) $(N=5$ plants per treatment $)$ using a CI-340 handheld photosynthesis system (CID-Bio-Sciences, Inc., 4845NW Camas Meadows Drive, Camas, WA, 98607, United States). Leaves were first equilibrated at $370 \mathrm{mmol} \mathrm{mol}{ }^{-1}$ of ambient $\mathrm{CO}_{2}$ and a photon density flux of $1,500 \mathrm{mmol} \mathrm{m}^{2} \mathrm{~s}^{-1}$ for at least 
$10 \mathrm{~min}$. Leaf temperature was maintained at $28^{\circ} \mathrm{C}$, with airflow set at $0.2 \mathrm{~L} \mathrm{~min}^{-1}$. These conditions were kept constant for the determination of $\mathrm{CO}_{2}$ assimilation rate (net photosynthesis), stomatal conductance, and transpiration.

\section{Antioxidant Enzymes}

Leaf tissue of all plant groups ( $N=3$ plants per treatment) was ground into a fine powder in liquid nitrogen and stored at $-80^{\circ} \mathrm{C}$ until protein extraction. The methodology for protein extraction and measurements of superoxide dismutase (SOD, EC 1.15.1), ascorbate peroxidase (APX, EC 1.11.1.11), peroxidase class III (POD, EC 1.11.1.7) and phenylalanine ammonia-lyase (PAL, EC 4.3.1.5) activity was previously described in Contreras et al. (2018, 2019). SOD activity was determined by using the photoinhibition of nitro-blue tetrazolium assay (NBT) at $560 \mathrm{~nm}$ of absorbance (Beauchamp and Fridovich, 1971). APX was measured based on the consumption of ascorbate at $290 \mathrm{~nm}$ of absorbance, according to Lima et al. (2002), using molar extinction of ascorbate, $\varepsilon=2.8 \mathrm{mM}^{-1} \mathrm{~cm}^{-1}$ (Köhler et al., 2017). POD activity was determined according to Pinhero et al. (1997), based on tetrahydro-guaiacol (THG) formation at $470 \mathrm{~nm}$ of absorbance using molar extinction of THG, $\varepsilon=26.6 \mathrm{mM}^{-1} \mathrm{~cm}^{-1}$ (Köhler et al., 2017). PAL activity was measured using the trans-cinnamic acid formation at $290 \mathrm{~nm}$, using d-phenylalanine as a negative control (Pellegrini et al., 1994).

\section{Phenolic Content}

Total phenolic content was determined using the Folin-Ciocalteu assay, as described in Contreras et al. (2018). The absorbance was measured at $660 \mathrm{~nm}$ in a microplate reader spectrophotometer (TECAN, Infinite2000pro, Austria) and expressed in gallic acid equivalents (GA) per gram of fresh weight (FW), following Singleton et al. (1999).

\section{Lipid Peroxidation}

Lipid peroxidation was estimated by measuring the concentration of malondialdehyde (MDA) by the thiobarbituric acid reactive substances (TBARS) assay. $50 \mathrm{mg}$ of fresh plant tissue was ground to a powder in liquid nitrogen and suspended in $1 \mathrm{~mL}$ of $1 \%$ trichloroacetic acid (TCA), and the homogenate centrifuged at $13,000 \mathrm{rpm}$ for $5 \mathrm{~min}$. $250 \mu \mathrm{L}$ of the supernatant was mixed with $1 \mathrm{~mL}$ of $0.5 \%$ thiobarbituric acid (TBA) in $20 \%$ TCA, and the mixture boiled for $30 \mathrm{~min}$ then cooled to room temperature. The MDA formed was quantified at $532 \mathrm{~nm}$ and $600 \mathrm{~nm}$ in a microplate reader spectrophotometer (TECAN, Infinite2000pro, Austria), using a molar extinction coefficient of $155 \mathrm{mM}^{-1} \mathrm{~cm}^{-1}$ (Ederli et al., 2004).

\section{Statistical Analysis}

Effects of fungal endophytes and salinity on physiological and biochemical traits were determined with a two-way ANOVA followed by a Tukey's HSD post hoc test. The presence of endophytes and salinity were considered fixed effects. Assumptions of normality and homogeneity of variance were tested prior to statistical analyses. All data followed these requirements except APX activity, which was log transformed to achieve homogeneity of variance. Analyses were performed using Statistica 7.0.

\section{RESULTS}

\section{Morphological and Physiological Responses}

Plant height was significantly affected by endophyte inoculation, salinity, and $\mathrm{E} \times \mathrm{S}$ interaction (Table 1). Plant height decreased in response to salinity in $\mathrm{E}$ - and $\mathrm{E} 2+$ plants $(P<0.05$, Tukey test), although it was not impacted by salinity in E1+ and E1E2+ plants (Figure 1A). The number of shoots was significantly affected by endophyte inoculation and by the interaction $\mathrm{E} \times \mathrm{S}$. Salinity did not significantly affect the number of shoots (Table 1). While the number of shoots decreased under salinity in E- plants $(P<0.05$, Tukey test $)$, it remained constant in inoculated plants (Figure 1B). Net photosynthesis was significantly affected by endophyte inoculation and salinity. No significant endophyte $\mathrm{x}$ salinity $(\mathrm{E} \times \mathrm{S})$ interaction effects were observed (Table 1). Photosynthesis was higher in E1E2+ plants than in E- and E2+ plants $(P<0.05$, Tukey test); nevertheless, no significant differences were observed between E1E2 + and E1+ plants ( $P>0.05$, Tukey test) (Figure 2A). While photosynthesis decreased considerably under salinity in E- plants, it was not affected by salinity in either single or coinoculated plants (Figure 2A). No significant effect of endophyte inoculation on stomatal conductance and transpiration was observed; however, both were significantly affected by salinity and $\mathrm{E} \times \mathrm{S}$ interaction. While stomatal conductance and transpiration declined in response to salinity in E- and E2+ plants, both remained constant in $\mathrm{E} 1+$ and E1E2+ plants (Figures 2B,C).

\section{Enzyme Activities}

SOD was significantly affected by endophyte inoculation, salinity, as well as an $\mathrm{E} \times \mathrm{S}$ interaction (Table 1). It was considerably

TABLE 1 | Two-way ANOVA of the effects of endophyte inoculation and salinity on physiological and biochemical traits of Chenopodium quinoa.

\begin{tabular}{|c|c|c|c|}
\hline & Endophyte (E) & Salinity (S) & $E \times S$ \\
\hline Plant height & $5.44^{\star \star}$ & $11.46^{\star *}$ & $6.32^{\star \star}$ \\
\hline Number of shoots & $3.29^{*}$ & $0.44 n s$ & $5.40^{\star *}$ \\
\hline Photosynthesis & $4.07^{*}$ & $6.69^{*}$ & $2.34 n s$ \\
\hline Stomatal conductance & $2.38 n s$ & $35.08^{\star \star \star}$ & $5.90^{\star *}$ \\
\hline Transpiration & $0.75 n s$ & $26.5^{\star \star \star}$ & $5.82^{\star \star}$ \\
\hline SOD & $46.1^{\star \star \star}$ & $59.8^{\star \star \star}$ & $11.0^{\star \star \star}$ \\
\hline APX & $146.4^{\star \star \star}$ & $14.8^{\star \star}$ & $16.2^{\star \star \star}$ \\
\hline POD & $123.3^{\star \star \star}$ & $81.8^{\star \star \star}$ & $16.3^{\star \star \star}$ \\
\hline PAL & $2.33 n s$ & $49.7^{\star \star \star}$ & $0.31 n s$ \\
\hline Phenolic content & $16.3^{\star \star \star}$ & $70.3^{\star \star \star}$ & $12.4^{\star \star \star}$ \\
\hline MDA & $64.8^{\star \star \star}$ & $0.47 n s$ & $3.69^{*}$ \\
\hline
\end{tabular}

F-ratios are shown. Significance levels are as follows: ${ }^{*} P<0.05,{ }^{* *} P<0.01$, ${ }^{* *} P<0.001 ; n s$ indicates no significant difference. $F$ ratios for physiological and morphological traits: $E=d f: 3.32, S=d f: 1.32, E \times S=d f: 3.32 . F$ ratios for biochemical traits: $E=3.16, S=1.16, E \times S 3.16$. 


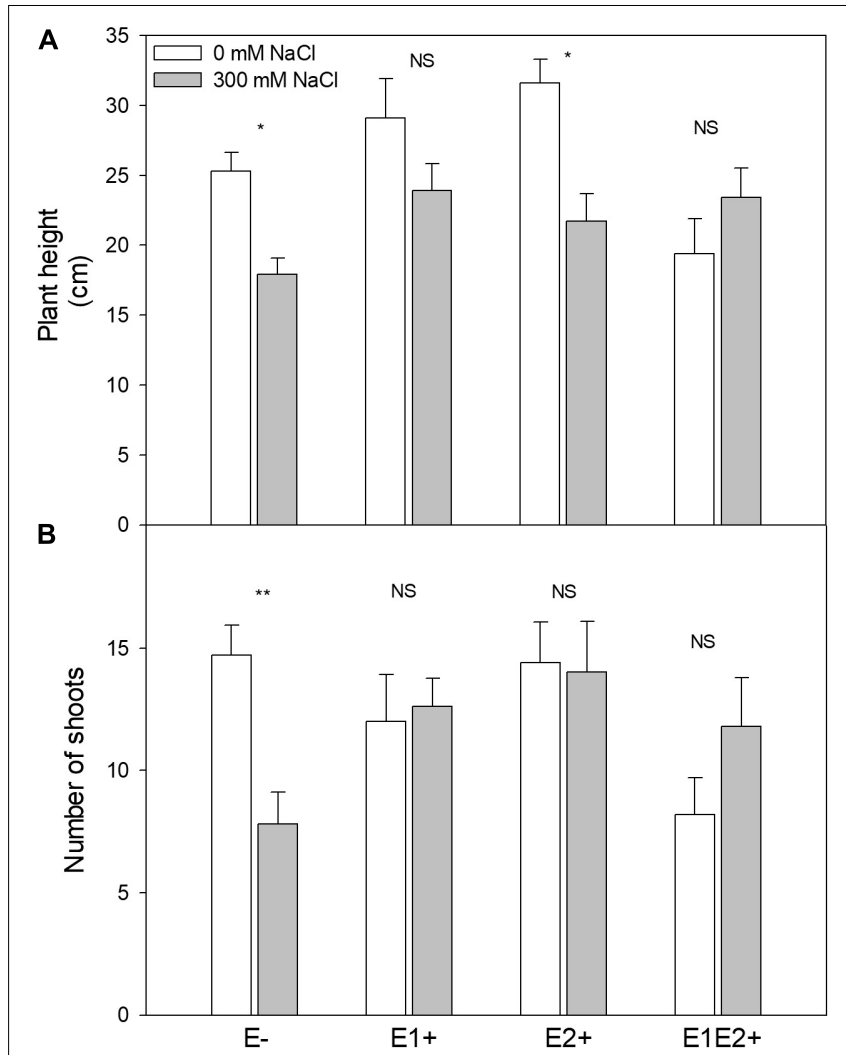

FIGURE 1 | Effects of fungal endophyte inoculation and salinity on plant height $(\mathbf{A})$ and number of shoots $(\mathbf{B})$ of Chenopodium quinoa. White bars = no salinity stress $(0 \mathrm{mM} \mathrm{NaCl})$; gray bars = salinity stress $(300 \mathrm{mM}$ $\mathrm{NaCl})$. E-: non-inoculated plants; E1+: plants inoculated with Talaromyces minioluteus; E2+: plants inoculated with Penicillium murcianum; E1E2+: plants inoculated with both endophytes in combination. Asterisks indicate the significance level of differences in plant responses between non-salt and salt treatments: ${ }^{* *} p<0.01,{ }^{*} p<0.05$, NS = no significant difference (Tukey HSD after two-way ANOVA).

higher in single- and co-inoculated plants than in non-inoculated plants $(P<0.05$, Tukey test) (Figure 3A). SOD activity increased under salinity in E-, E2+ and E1E2+ plants. For E1+ plants, however, there was no difference in SOD between control and saline treated plants (Figure 3A). Both APX and POD were significantly affected by endophyte inoculation, salinity, and $\mathrm{E} \times \mathrm{S}$ interaction (Table 1). Enzyme activity of both was greater in $\mathrm{E} 2+$ and $\mathrm{E} 1 \mathrm{E} 2+$ plants in comparison to $\mathrm{E}$ and $\mathrm{E} 1+$ plants $(P<0.05$, Tukey test) (Figures 3B,C). APX and POD decreased in response to salinity in E- and E2+ plants but remained constant under salinity in E1+ and in E1E2+ plants (Figures 3B,C). PAL was significantly affected by salinity (Table 1); nevertheless, no significant effects of endophyte inoculation or an $\mathrm{E} \times \mathrm{S}$ interaction were observed. PAL increased under salinity in all treatments (Figure 3D).

\section{Phenolic Content}

Total phenolic content was significantly affected by endophyte inoculation, salinity, and E x S interaction (Table 1). Phenolic content was higher in E1E2+ plants than in E- plants $(P<0.05$,

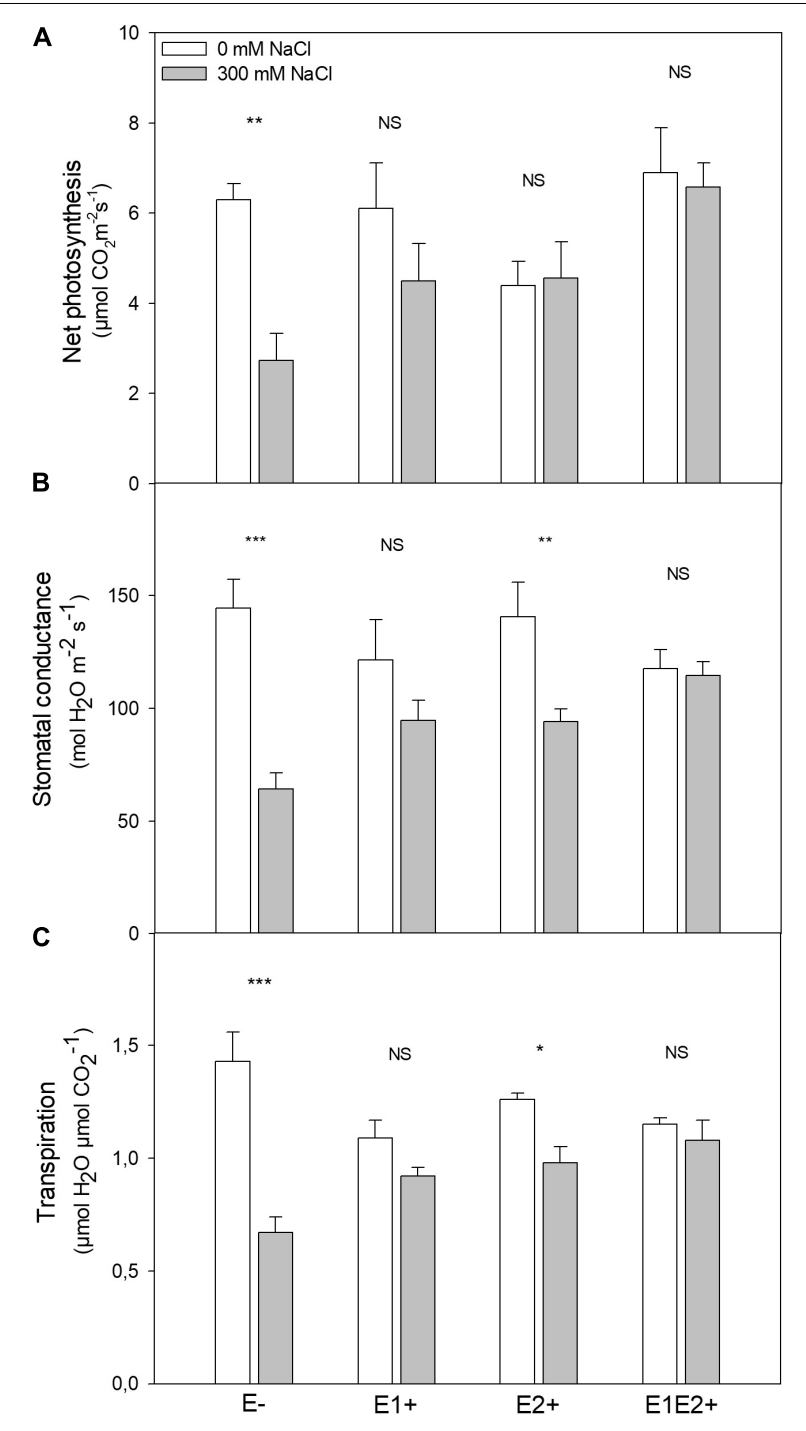

FIGURE 2 | Effects of fungal endophyte inoculation and salinity on net photosynthesis (A), stomatal conductance (B), and transpiration (C) of Chenopodium quinoa. White bars = no salinity stress (0 mM NaCl); gray bars = salinity stress (300 mM NaCl). E-: non-inoculated plants; E1+: plants inoculated with Talaromyces minioluteus; E2+: plants inoculated with Penicillium murcianum; E1E2+: plants inoculated with both endophytes in combination. Asterisks indicate the significance level of differences in plant responses between non-salt and salt treatments: ${ }^{* \star *} p<0.001,{ }^{\star \star} p<0.01$, ${ }^{*} p<0.05, \mathrm{NS}=$ no significant difference (Tukey HSD after two-way ANOVA).

Tukey test) (Figure 4), but no significant differences in its content were found between single- and co-inoculated plants $(P>0.05$, Tukey test). The phenolic content increased under salinity in E- and E1E2+ plants; nevertheless, it was not different between control and saline treated plants for groups E1+ and E2+ (Figure 4).

\section{Lipid Peroxidation}

Endophyte inoculation and $\mathrm{E} \times \mathrm{S}$ interaction significantly affected MDA level, but no significant effect was observed for 
salinity (Table 1). MDA was considerably lower in single- and in co-inoculated plants relative to non-inoculated plants $(P<0.05$, Tukey test) (Figure 5). No differences in MDA were observed between control and salinity for any plant group (Figure 5).

\section{DISCUSSION}

\section{Benefits of Fungal Endophytes in Plant Performance}

We showed that FE species isolated from quinoa growing in the Atacama Desert were able to mitigate negative effects of salinity in plants, suggesting a mutualistic relationship between $C$. quinoa and both fungal species under stress conditions. Both genera Talaromyces and Penicillium, however, are common soil inhabitants with different lifestyles (Visagie et al., 2014; Yilmaz et al., 2016). In this case, species of both genera may behave as endophytes, saprophytes or even as pathogens for some plant species (Visagie et al., 2014; Pasricha et al., 2017; Vinale et al., 2017; Tannous et al., 2020). In C. quinoa, FE promoted morphological, physiological, and biochemical responses. Morphological performance as well as photosynthesis were considerably improved in both single and co-inoculated plants. Stomatal conductance and transpiration, however, showed no improvement in response to FE. This suggests that other processes unrelated to gas exchange, such as enzyme and non-enzyme antioxidant mechanisms, could be involved in these responses. We showed that in quinoa antioxidant mechanisms, including SOD, APX, POD, and phenols, were enhanced by FE inoculation. SOD and APX are present in a range of organelles, including chloroplasts, and play a role in the water-water cycle, which is involved in ROS prevention (Asada, 1999). Whereas SOD catalyzes the dismutation of superoxide radicals into hydrogen peroxide $\left(\mathrm{H}_{2} \mathrm{O}_{2}\right)$ and molecular oxygen $\left(\mathrm{O}^{2}\right)$ downstream, APX catalyzes the reaction between ascorbic acid and $\mathrm{H}_{2} \mathrm{O}_{2}$ to produce water and monodehydroascorbic acid (Asada, 1999; Maruta et al., 2016). While loss of SOD isoforms has been demonstrated to lead to inactivation of photosystem I and increase photooxidative stress in plants, overexpression of this enzyme seems to result in an improvement in photosynthesis (Myouga et al., 2008; Gallie and Chen, 2019). Additionally, APX influences the $\mathrm{H}_{2} \mathrm{O}_{2}$ signaling mechanism related to stress responses and has been shown to lead to an improvement in the recovery of photosynthesis subsequent to plant stress (Uchida et al., 2002; Sales et al., 2013; Exposito-Rodriguez et al., 2017). Considering the role of both enzymes in photoprotection (Asada, 1999), FE inoculation in C. quinoa may promote photosynthesis, likely mediated by ROS reduction. These potentially positive effects of SOD and APX in quinoa, preventing photooxidative damage and improving photosynthetic responses, should be further assessed by measuring the photochemical balance through chlorophyll $a$ fluorescence analysis (Kramer et al., 2004). In addition to SOD and APX, POD (usually present in cell walls) and phenols play a key role scavenging $\mathrm{H}_{2} \mathrm{O}_{2}$ in plants (Lin and Kao, 2001; Blokhina et al., 2003). Whereas POD was found to be similarly induced as $\mathrm{APX}$ by FE in C. quinoa, the phenolic content was considerably improved by FE inoculation. Importantly, enhanced antioxidant systems (enzymatic and non-enzymatic) mediated by FE in quinoa were observed to lead to low levels of lipid peroxidation. Our results suggest that both $T$. minioluteus and $P$. murcianum assist $C$. quinoa in alleviating ROS production, which contributes to counteracting oxidative stress under stressful conditions (Singh et al., 2011; Gupta et al., 2021).

\section{Fungal Endophytes and Plant Tolerance to Salinity}

In the absence of stress, co-inoculation had a negative impact on morphological responses in C. quinoa compared to single and non-inoculation. In symbiotic associations, such as plantmycorrhiza or plant-FE interactions, the fungus acquires carbon from the plant, with possible impacts on plant growth (Behie et al., 2017). Our results suggest that multiple symbiosis likely acquires more carbohydrates from the plant than single symbiosis. Conversely, under conditions of stress, co-inoculation allowed growth to remain constant relative to non-inoculated plants, in which morphological traits were negatively affected by salinity. Salinity negatively impacted morphological responses in E-; nevertheless, they remained stable under salinity in single and co-inoculated plants. A similar pattern was observed for photosynthesis, which suggests that positive FE effects on photosynthesis in C. quinoa resulted in improved plant biomass. Our results are consistent with a previous study on C. quinoa, which showed that benefits of plant-endophyte associations seem to be more pronounced under conditions of stress (GonzálezTeuber et al., 2017). The ability of both fungal genera to confer salinity tolerance to C. quinoa is likely a result of an evolutionary adaptation of the former to cope with stressful environments (dry and saline conditions), which is ultimately passed on to the host (Rodriguez et al., 2008).

Salt stress may result in degradation of photosynthetic pigments as well as D1 and D2 proteins of the photoreaction center, which leads to a decline in photosynthesis (Jansen et al., 1996). Here, we showed that salinity considerably impaired photosynthesis in non-inoculated plants, which was coupled with a reduction in stomatal conductance and transpiration. In contrast, endophyte inoculation allowed $C$. quinoa to keep photosynthesis constant in response to salinity, attaining levels similar to those observed in non-inoculated plants under control conditions. This is likely promoted by maintenance of stomatal conductance and transpiration, suggesting that FE regulate stomatal opening, mitigating negative effects of salinity in C. quinoa. Similar responses to salinity stress have been observed in other FE-inoculated crop species (Jogawat et al., 2013; Molina-Montenegro et al., 2020). In rice seedlings, for example, it was found that the root-endophytic fungus Piriformospora indica triggered an increase in photosynthetic pigment content under salinity stress relative to non-inoculated salt-treated rice seedlings, which showed a decline in these pigments (Jogawat et al., 2013). FE may mitigate salinity effects on photosynthesis by improving plant water status, which may result in greater stomatal conductance and ultimately higher $\mathrm{CO}_{2}$ assimilation (Zarea et al., 2012). Several studies have 


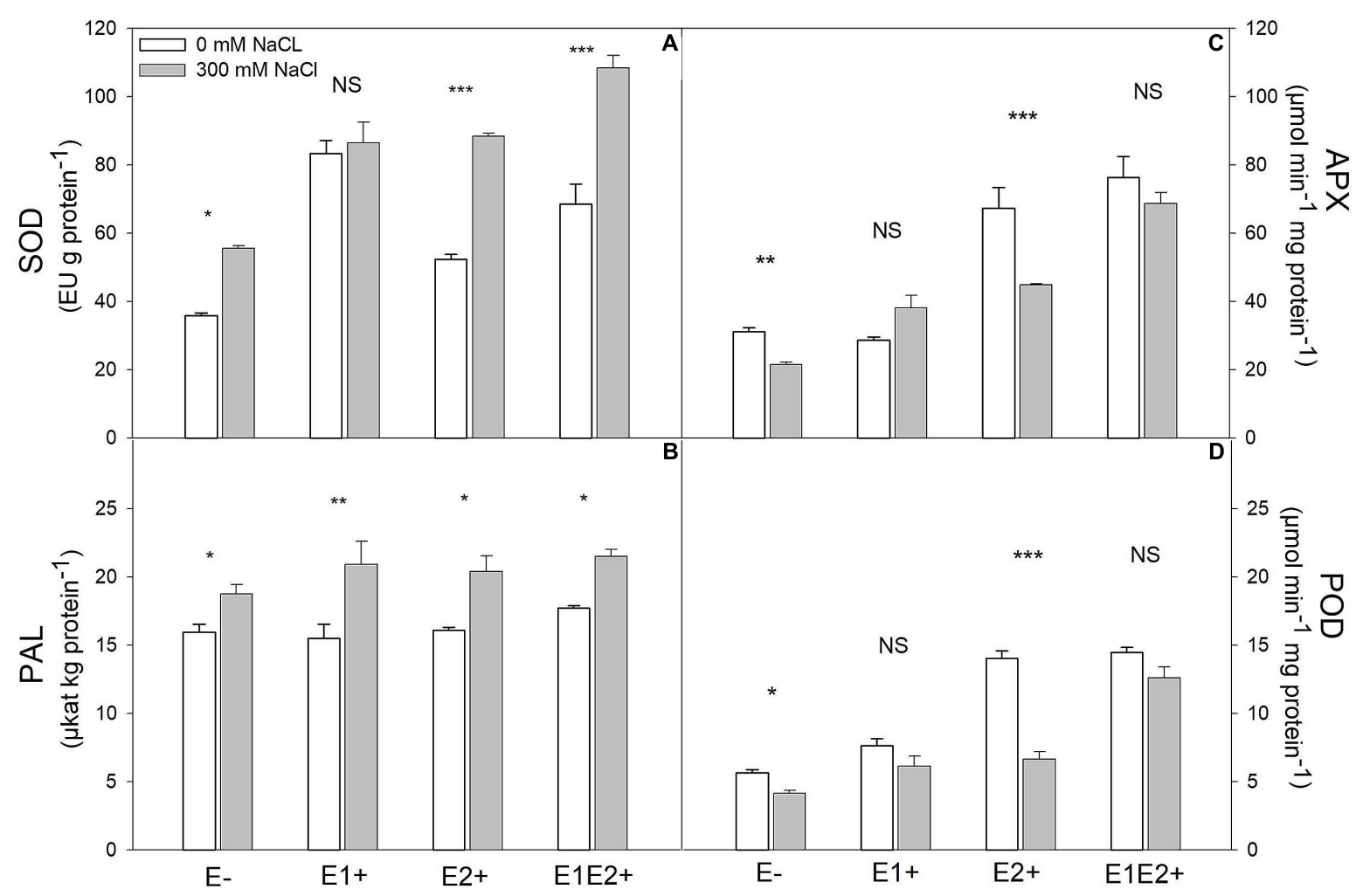

FIGURE 3 | Effects of fungal endophyte inoculation and salinity on antioxidant enzyme activities: Superoxide dismutase (SOD) (A), ascorbate peroxidase (APX) (B), peroxidase (POD) (C), and phenylalanine ammonia-lyase (PAL) (D) of Chenopodium quinoa. White bars = no salinity stress (0 mM NaCl); gray bars = salinity stress (300 mM NaCl). E-: non-inoculated plants; E1+: plants inoculated with Talaromyces minioluteus; E2+: plants inoculated with Penicillium murcianum; E1E2+: plants inoculated with both endophytes in combination. Asterisks indicate the significance level of differences in plant responses between non-salt and salt treatments: ${ }^{\star \star \star} p<0.001,{ }^{\star \star} p<0.01,{ }^{\star} p<0.05, \mathrm{NS}=$ no significant difference (Tukey HSD after two-way ANOVA).

reported activation of antioxidant systems mediated by $\mathrm{FE}$ under salinity stress (Bagheri et al., 2013; Radhakrishnan et al., 2013; Li et al., 2017; Zhang et al., 2019). In C. quinoa, SOD activity increased in response to salinity in non-inoculated and inoculated plants. SOD improvement under saline conditions is a common response in a wide range of plants (including C. quinoa), even in the absence of fungal symbiosis (Cai and Gao, 2020). Nevertheless, FE inoculation, and particularly coinoculation, improved SOD activity compared to non-inoculated plants. APX and POD have often been observed to increase under saline conditions in FE inoculated plants (Bagheri et al., 2013; Li et al., 2017; Zhang et al., 2019), although this was not the case in our study; rather, they remained constant in saline treated groups $\mathrm{E} 2+$ and E1E2+. This suggests that these enzymes are unlikely to play a significant role in C. quinoa in response to salinity. Both enzymes are likely important in the absence of stress in quinoa, however, showing a strong activation response to FE inoculation under non-saline treatments. Additionally, we found that both PAL and phenolic content increased in response to salinity, which is expected, since PAL is involved in the phenylpropanoid pathway, which leads to the synthesis of secondary metabolites such as phenolic compounds (Hahlbrock and Scheel, 1989). Taken together, our results showed that FE assist C. quinoa to induce both antioxidant enzymes and metabolites, improving plant tolerance to salinity stress. Salinity usually increases the level of lipid peroxidation in plants (Al Hassan et al., 2017), which may lead to higher membrane permeability and ion loss from the cells (Gupta and Huang, 2014). Here, we showed that salinity did not elevate MDA content in either inoculated or non-inoculated quinoa plants. This suggests an innate tolerance to salinity in the C. quinoa cultivar used in this study.

\section{Synergic Fungal Endophyte Association and Antioxidative System}

Our results showed that, compared to single-inoculated plants, co-inoculated C. quinoa individuals were better able to mitigate salinity stress. Co-inoculation improved photosynthesis and antioxidant mechanisms, including SOD, APX, POD, and phenolic content. This is consistent with previous studies, which have revealed synergistic effects of a range of microbes on hosts, increasing plant biomass and tolerance to abiotic stresses, including drought, salinity, and heat (Larimer et al., 2014; Bilal et al., 2020; Moreira et al., 2020). The mechanisms underlying host oxidative stress protection by FE are attributed mainly to the production of numerous antioxidant compounds in plant tissues, including 


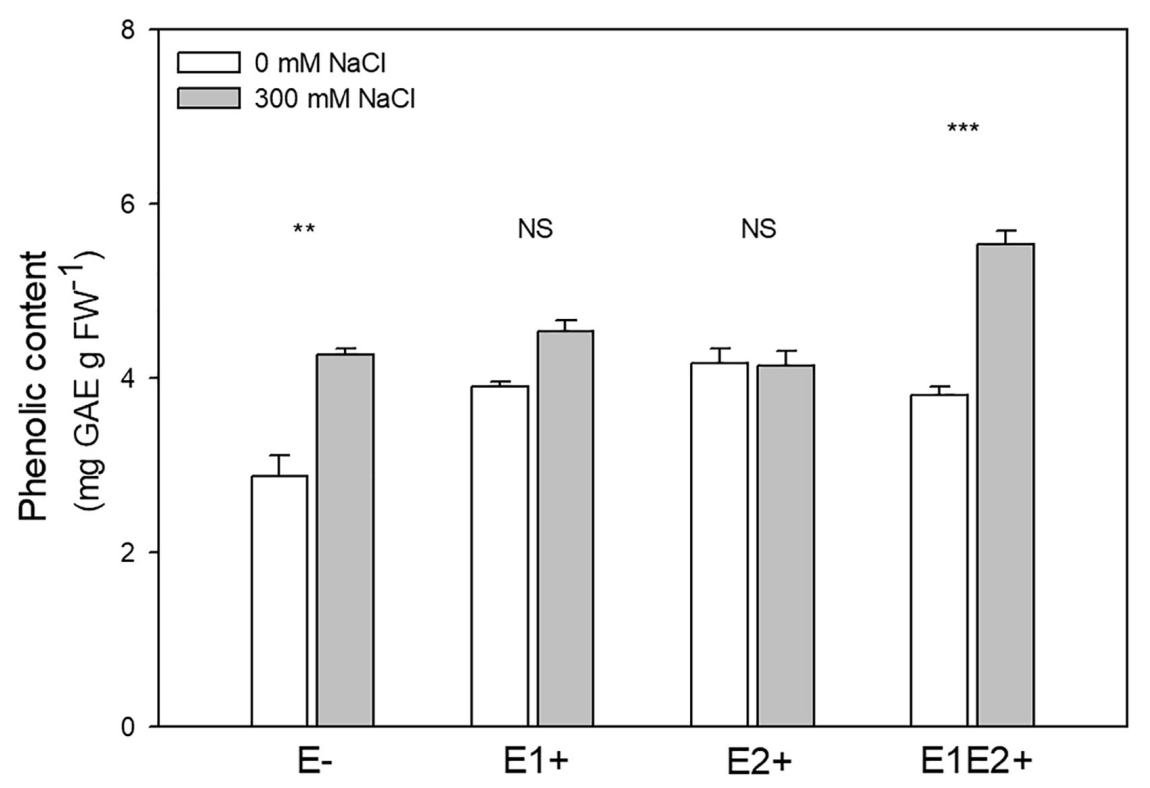

FIGURE 4 | Effects of fungal endophyte inoculation and salinity on phenolic content of Chenopodium quinoa. White bars = no salinity stress $(0 \mathrm{mM}$ NaCl); gray bars = with salinity stress (300 mM NaCl). E-: non-inoculated plants; E1+: plants inoculated with Talaromyces minioluteus; E2+: plants inoculated with Penicillium murcianum; E1E2+: plants inoculated with both endophytes in combination. Asterisks indicate the significance level of differences in plant responses between non-salt and salt treatments: ${ }^{* * *} p<0.001,{ }^{* *} p<0.01, \mathrm{NS}=$ no significant difference (Tukey HSD after two-way ANOVA).

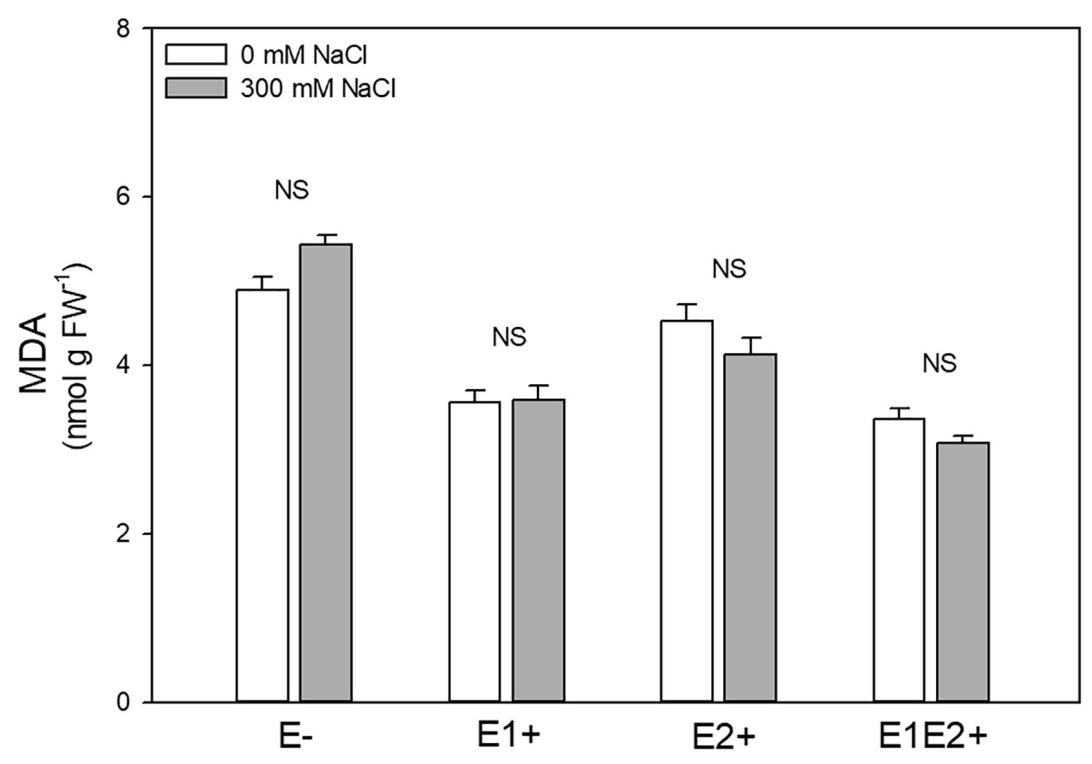

FIGURE 5 | Effects of fungal endophyte inoculation and salinity on malondialdehyde (MDA, product of lipid peroxidation) of Chenopodium quinoa. White bars = no salinity stress (0 mM NaCl); gray bars = salinity stress (300 mM NaCl). E-: non-inoculated plants; E1+: plants inoculated with Talaromyces minioluteus; E2+: plants inoculated with Penicillium murcianum; E1E2+: plants inoculated with both endophytes together. NS = no significant difference (Tukey HSD after two-way ANOVA).

alkaloids, flavonoids, phenolic acids, and antioxidant enzymes (Huang et al., 2007; Hamilton et al., 2012; Gul Jan et al., 2019), which are regulated by environmental conditions (Pusztahelyi et al., 2015). Huang et al. (2007), for example, examined 292 FE isolated from a diverse range of plants and measured antioxidant and phenolic production, suggesting that all FE are able to produce antioxidant and phenolic compounds themselves. Further, the FE Yarrowia lipolytica synthesizes in vitro polyphenols and flavonoids in appreciable quantities. Additionally, inoculation of maize by this species resulted in improved plant physiological and biochemical responses to salinity (Gul Jan et al., 2019). Since FE communities are diverse 
and may produce a mixture of bioactive metabolites, including stress protective compounds, it is suggested that more complex microbial communities would be more likely to improve host tolerance to environmental stresses (Van Elsas et al., 2012; Bilal et al., 2020; Moreira et al., 2020). Given that FE are able to alter host chemical profiles (Markert et al., 2008; Gul Jan et al., 2019), it is expected that these antioxidant compounds are likely part of the strategy of FE to deal with oxidative stress in plant tissues. FE play an important ecological role in the ability of some plant species to grow under extreme environmental conditions (Rodriguez et al., 2004, 2008), which is likely attributable to endophyte antioxidants that contribute to enhancing overall stress tolerance in plants. Some studies have proposed that a balance between elevated ROS and antioxidant production during plant-fungus symbiosis might be an additional mechanism explaining beneficial effects conferred by FE to plants inhabiting stressful environments (White and Torres, 2010; Hamilton et al., 2012). Since both partners (FE and plants) must cope with stress-induced ROS, a plausible result is the production of antioxidants by both partners in the symbiosis (White and Torres, 2010). This would imply a mutually enhanced protection to oxidative stress. For the C. quinoa-Talaromyces/Penicillium interaction, further studies are needed to elucidate the chemical basis of the symbiotic communication responsible for plant stress tolerance.

\section{CONCLUSION}

In C. quinoa, benefits provided by FE under saline conditions included an induction of antioxidant enzymes and antioxidant metabolites, improving photosynthesis and plant performance. Additional mechanisms potentially involved in mitigation of salt effects on plant performance by $\mathrm{FE}$ in $C$. quinoa are still unknown and require further investigation. Antioxidant compounds secreted by the plant-fungus symbiosis under stressful conditions would contribute to counteracting salinityinduced oxidative stress in C. quinoa. Moreover, our study highlights the importance of multiple microbial symbionts acting in tandem to increase host benefits. Future research in this

\section{REFERENCES}

Acuña-Rodríguez, I. S., Hansen, H., Gallardo-Cerda, J., Atala, C., and MolinaMontenegro, M. (2019). Antarctic extremophiles: biotechnological alternative to crop productivity in saline soils. Front. Bioeng. Biotechnol. 7:22. doi: 10.3389/ fbioe.2019.00022

Al Hassan, M., Chaura, J., Donat-Torres, M. P., Boscaiu, M., and Vicente, O. (2017). Antioxidant responses under salinity and drought in three closely related wild monocots with different ecological optima. AoB Plants 9:lx009. doi: 10.1093/ aobpla/plx009

Apel, K., and Hirt, H. (2004). Reactive oxygen species: metabolism, oxidative stress, and signal transduction. Annu. Rev. Plant Biol. 55, 373-399. doi: 10.1146/ annurev.arplant.55.031903.141701

Arnold, A. E., Maynard, Z., Gilbert, G. S., Coley, P. D., and Kursar, T. A. (2000). Are tropical fungal endophytes hyperdiverse? Ecol. Lett. 3, 267-274. doi: 10.1046/j. 1461-0248.2000.00159.x

Asada, K. (1999). The water-water cycle in chloroplasts: scavenging of active oxygens and dissipation of excess photons. Annu. Rev. Plant Physiol. Plant Mol. Biol. 50, 601-639. doi: 10.1146/annurev.arplant.50.1.601 context should consider testing the effects of Talaromyces and Penicillium species on other crop species to evaluate potential benefits to agricultural production systems.

\section{DATA AVAILABILITY STATEMENT}

The raw data supporting the conclusions of this article will be made available by the authors, without undue reservation.

\section{AUTHOR CONTRIBUTIONS}

MG-T designed the research and analyzed the data. MG-T and DB carried out the experiment. DB, LB-G, RC, and GZ developed measurements and analyses. MG-T and LB-G wrote the manuscript. All authors contributed to the article and approved the submitted version.

\section{FUNDING}

This work was supported by the UCSC-MPS Partner Group (MG-T), ANID-Max Planck Society Grant, MPG190015 (MG-T), and ANID Fondecyt Regular No. 1211473 (LB-G).

\section{ACKNOWLEDGMENTS}

We thank María José Guevara for her valuable help in the lab. We are grateful to the Socaire Community for its generous contribution with quinoa seeds. MG-T acknowledges the Max Planck Society (MPS) through the Max Planck Partner Group.

\section{SUPPLEMENTARY MATERIAL}

The Supplementary Material for this article can be found online at: https://www.frontiersin.org/articles/10.3389/fevo.2021. 787318/full\#supplementary-material

Atlas, R., and Parks, L. C. (1993). Handbook of Microbiological Media. Boca Raton, FL: CRC Press.

Azad, K., and Kaminskyj, S. (2016). A fungal endophyte strategy for mitigating the effect of salt and drought stress on plant growth. Symbiosis 68, 73-78. doi: 10.1007/s13199-015-0370-y

Bagheri, A. A., Saadatmand, S., Niknam, V., Nejadsatari, T., and Babaeizad, V. (2013). Effect of endophytic fungus, Piriformospora indica, on growth and activity of antioxidant enzymes of rice (Oryza sativa L.) under salinity stress. Int. J. Adv. Biol. Biomed. Res. 1, 1337-1350.

Baltruschat, H., Fodor, J., Harrach, B. D., Niemczyk, E., Barna, B., Gullner, G., et al. (2008). Salt tolerance of barley induced by the root endophyte Piriformospora indica is associated with a strong increase in antioxidants. New Phytol. 180, 501-510. doi: 10.1111/j.1469-8137.2008.02583.x

Bartels, D., and Sunkar, R. (2005). Drought and salt tolerance in plants. Crit. Rev. Plant Sci. 24, 23-58. doi: 10.1080/07352680590910410

Bascuñán-Godoy, L., Reguera, M., Blumwald, Y. M., and Blumwald, E. (2016). Water deficit stress-induced changes in carbon and nitrogen partitioning in Chenopodium quinoa Willd. Planta 243, 591-603. doi: 10.1007/s00425-0152424-z 
Beauchamp, C., and Fridovich, I. (1971). Superoxide dismutase: improved assays and an assay applicable to acrylamide gels. Anal. Biochem. 44, 276-287. doi: 10.1016/0003-2697(71)90370-8

Behie, S. W., Moreira, C. C., Sementchoukova, I., Barelli, L., Zelisko, P. M., and Bidochka, M. J. (2017). Carbon translocation from a plant to an insect-pathogenic endophytic fungus. Nat. Commun. 8:14245. doi: 10.1038/ ncomms 14245

Bhargava, A., Shukla, S., and Ohri, D. (2006). Chenopodium quinoa-an Indian perspective. Ind. Crops Prod. 23, 73-87. doi: 10.1016/j.indcrop.2005.04.002

Bilal, S., Shazad, R., Imran, M., Jan, R., Min Kim, K., and Lee, I.-J. (2020). Synergistic association of endophytic fungi enhances Glycine max L. resilience to combined abiotic stresses: heavy metals, high temperature and drought stress. Ind. Crops Prod. 143:111931. doi: 10.1016/j.indcrop.2019.111931

Blokhina, O., Virolainen, E., and Fagerstedt, K. V. (2003). Antioxidants, oxidative damage and oxygen deprivation stress: a review. Ann. Bot. 91, 179-194. doi: $10.1093 / \mathrm{aob} / \mathrm{mcf} 118$

Bose, J., Rodrigo-Moreno, A., and Shabala, S. (2014). ROS homeostasis in halophytes in the context of salinity stress tolerance. J. Exp. Bot. 65, 1241-1257. doi: $10.1093 /$ jxb/ert430

Cai, Z.-Q., and Gao, Q. (2020). Comparative physiological and biochemical mechanisms of salt tolerance in five contrasting highland quinoa cultivars. $B M C$ Plant Biol. 20:70. doi: 10.1186/s12870-020-2279-8

Contreras, R. A., Pizarro, M., Köhler, H., Sáez, C. A., and Zúñiga, G. E. (2018). Copper stress induces antioxidant responses and accumulation of sugars and phytochelatins in Antarctic Colobanthus quitensis (Kunth) Bartl. Biol. Res. 51:48. doi: 10.1186/s40659-018-0197-0

Contreras, R. A., Pizarro, M., Köhler, H., Zamora, P., and Zúñiga, G. E. (2019). UV-B shock induces photoprotective flavonoids but not antioxidant activity in Antarctic Colobanthus quitensis (Kunth) Bartl. Environ. Exp. Bot. 159, 179-190. doi: 10.1016/j.envexpbot.2018.12.022

Ederli, L., Reale, L., Ferranti, F., and Pasqualini, S. (2004). Responses induced by high concentration of cadmium in Phragmites australis roots. Physiol. Plant. 121, 66-74. doi: 10.1111/j.0031-9317.2004.00295.x

Exposito-Rodriguez, M., Laissue, P. P., Yvon-Durocher, G., Smirnoff, N., and Mullineaux, P. M. (2017). Photosynthesis-dependent $\mathrm{H}_{2} \mathrm{O}_{2}$ transfer from chloroplasts to nuclei provides a high-light signaling mechanism. Nat. Commun. 8:49. doi: 10.1038/s41467-017-00074-w

Foyer, C. H., and Noctor, G. (2003). Redox sensing and signalling associated with reactive oxygen in chloroplasts, peroxisomes and mitochondria. Physiol. Plant. 119, 355-364. doi: 10.1034/j.1399-3054.2003.00223.x

Fuentes, F., and Bhargava, A. (2011). Morphological analysis of quinoa germplasm grown under lowland desert conditions. J. Agron. Crop Sci. 197, 124-134. doi: 10.1111/j.1439-037x.2010.00445.x

Gallie, D. R., and Chen, Z. (2019). Chloroplast-localized iron superoxide dismutases FSD2 and FSD3 are functionally distinct in Arabidopsis. PLoS One 14:e0220078. doi: 10.1371/journal.pone.0220078

García, M., Raes, D., Jacobsen, S. E., and Michel, T. (2007). Agroclimatic constraints for rainfed agriculture in the Bolivian Altiplano. J. Arid Environ. 71, 109-121. doi: 10.1016/j.jaridenv.2007.02.005

Gazis, R., and Chaverri, P. (2015). Wild trees in the Amazon basin harbor a great diversity of beneficial endosymbiotic fungi: is this evidence of protective mutualism? Fungal Ecol. 17, 18-29. doi: 10.1016/j.funeco.2015.04.001

Giauque, H., Connor, E. W., and Hawkes, C. V. (2018). Endophyte traits relevant to stress tolerance, resource use and habitat of origin predict effects on host plants. New Phytol. 221, 2239-2249. doi: $10.1111 /$ nph. 15504

González-Teuber, M. (2016). The defensive role of foliar endophytic fungi for a South American tree. AoB Plants 8:1w050. doi: 10.1093/aobpla/plw050

González-Teuber, M., Urzúa, A., Plaza, P., and Bascuñán-Godoy, L. (2018). Effects of root endophytic fungi on response of Chenopodium quinoa to drought stress. Plant Ecol. 219, 231-240. doi: 10.1007/s11258-017-0791-1

González-Teuber, M., Vilo, C., and Bascuñán-Godoy, L. (2017). Molecular characterization of endophytic fungi associated with the roots of Chenopodium quinoa inhabiting the Atacama Desert, Chile. Genom. Data 11, 109-112. doi: 10.1016/j.gdata.2016.12.015

Gul Jan, F., Hamayun, M., Hussain, A., Jan, G., Iqbal, A., Khan, A., et al. (2019). An endophytic isolate of the fungus Yarrowia lipolytica produces metabolites that ameliorate the negative impact of salt stress on the physiology of maize. BMC Microbiol. 19:3. doi: 10.1186/s12866-018-1374-6

Gupta, B., and Huang, B. (2014). Mechanism of salinity tolerance in plants: physiological, biochemical, and molecular characterization. Int. J. Genom. 2014, 701596-701596. doi: 10.1155/2014/701596

Gupta, S., Schillaci, M., Walker, R., Smith, P. M. C., Watt, M., and Roessner, U. (2021). Alleviation of salinity stress in plants by endophytic plant-fungal symbiosis: current knowledge, perspectives and future directions. Plant Soil 461, 219-244. doi: 10.1007/s11104-020-04618-w

Hahlbrock, K., and Scheel, D. (1989). Physiology and molecular biology of phenylpropanoid metabolism. Annu. Rev. Plant Physiol. Plant Mol. Biol. 4, 347-369. doi: 10.1146/annurev.pp.40.060189.002023

Hamilton, C. E., Gundel, P. E., Helander, M., and Saikkonen, K. (2012). Endophytic mediation of reactive oxygen species and antioxidant activity in plants: a review. Fungal Divers. 54, 1-10. doi: 10.1007/s13225-012-0158-9

Hnilickova, H., Kraus, K., Vachova, P., and Hnilicka, F. (2021). Salinity stress affects photosynthesis, malondialdehyde formation, and proline content in Portulaca oleraceae. Plants 10:845. doi: 10.3390/plants10050845

Houston, J., and Hartley, A. J. (2003). The central Andean westslope rainshadow and its potential contribution to the origin of hyperaridity in the Atacama Desert. Int. J. Climatol. 23, 1453-1464. doi: 10.1002/joc.938

Huang, W.-Y., Cai, Y.-Z., Xing, J., Corke, H., and Sun, M. (2007). A potential antioxidant resource: endophytic fungi from medicinal plants. Econ. Bot. 61, 14-30.

Hussain, M. I., Muscolo, A., Ahmed, M., Asghar, M. A., and AlDakheel, A. J. (2020). Agro-morphological, yield and quality traits and interrelationship with yield stability in quinoa (Chenopodium quinoa Willd.) genotypes under saline marginal environment. Plants 9:1763. doi: $10.3390 /$ plants9121763

Jansen, M. A., Gaba, V., Greenberg, B. M., Mattoo, A. K., and Edelman, M. (1996). Low threshold levels of ultraviolet-B in a background of photosynthetically active radiation trigger rapid degradation of the D2 protein of photosystem-II. Plant J. 9, 693-699. doi: 10.1046/j.1365-313X.1996.9050693.x

Jaramillo Roman, V., den Toom, L. A., Castro Gamiz, C., van der Pijl, N., Visser, R. G. F., van Loo, E. N., et al. (2020). Differential responses to salt stress in ion dynamics, growth and seed yield of European quinoa varieties. Environ. Exp. Bot. 177:104146. doi: 10.1016/j.envexpbot.2020.104146

Jogawat, A., Saha, S., Bakshi, M., Dayaman, V., Kumar, M., Dua, M., et al. (2013). Piriformospora indica rescues growth diminution of rice seedlings during high salt stress. Plant Signal. Behav. 8:e26891.

Köhler, H., Contreras, R. A., Pizarro, M., Cortés-Antíquera, R., and Zúñiga, G. E. (2017). Antioxidant responses induced by UVB radiation in Deschampsia antarctica desv. Front. Plant Sci. 8:921. doi: $10.3389 /$ fpls.2017.00921

Kramer, D. M., Johnson, G., Kiirats, O., and Edwards, G. E. (2004). New fluorescence parameters for the determination of QA redox state and excitation energy fluxes. Photosynth. Res. 79, 209-218. doi: 10.1023/B:PRES.0000015391. 99477.0d

Larimer, A. L., Clay, K., and Bever, J. D. (2014). Synergism and context dependency of interactions between arbuscular mycorrhizal fungi and rhizobia with prairie legume. Ecology 95, 1045-1054. doi: 10.1890/13-0025.1

Li, L., Li, L., Wang, X., Zhu, P., Wu, H., and Qi, S. (2017). Plant growth promoting endophyte Piriformospora indica alleviates salinity stress in Medicago truncatula. Plant Physiol. Biochem. 119, 211-223. doi: 10.1016/j. plaphy.2017.08.029

Lima, A. L. S., DaMatta, F. M., Pinheiro, H. A., Totola, M. R., and Loureiro, M. E. (2002). Photochemical responses and oxidative stress in two clones of Coffea canephora under water deficit conditions. Environ. Exp. Bot. 47, 239-247. doi: 10.1016/S0098-8472(01)00130-7 
Lin, C. C., and Kao, C. H. (2001). Cell wall peroxidase activity, hydrogen peroxide level and NaCl-inhibited root growth of rice seedlings. Plant Soil 230, 135-143. doi: 10.1023/A:1004876712476

Lutz, M., and Bascuñán-Godoy, L. (2017). "The revival of quinoa: a crop for health," in Superfood and Functional Food-An Overview of Their Processing and Utilization, eds V. Waisundara and N. Shiomi (London: InTech), $37-54$.

Markert, A., Steffan, N., Ploss, K., Hellwig, S., Steiner, U., Drewke, C., et al. (2008). Biosynthesis and accumulation of ergoline alkaloids in a mutualistic association between Ipomoea asarifolia (Convolvulaceae) and a clavicipitalean fungus. Plant Physiol. 147, 296-305. doi: 10.1104/pp.108.116699

Maruta, T., Sawa, Y., Shigeoka, S., and Ishikawa, T. (2016). Diversity and evolution of ascorbate peroxidase functions in chloroplasts: more than just a classical antioxidant enzyme? Plant Cell Physiol. 57, 1377-1386. doi: 10.1093/pcp/ pcv203

Meloni, D. A., Oliva, M. A., Martinez, C. A., and Cambraia, J. (2003). Photosynthesis and activity of superoxide dismutase, peroxidase and glutathione reductase in cotton under salt stress. Environ. Exp. Bot. 49, 69-76. doi: 10.1016/S0098-8472(02)00058-8

Moghaddam, M. S. H., Safaie, N., Soltani, J., and Hagh-Doust, N. (2021). Desertadapted fungal endophytes induce salinity and drought stress resistance in model crops. Plant Physiol. Biochem. 160, 225-238. doi: 10.1016/j.plaphy.2021. 01.022

Molina-Montenegro, M. A., Acuña-Rodríguez, I. S., Torres-Díaz, C., Gundel, P. E., and Dreyer, I. (2020). Antarctic root endophytes improve physiological performance and yield in crops under salt stress by enhanced energy production and $\mathrm{Na}^{+}$sequestration. Sci. Rep. 10:5819. doi: 10.1038/s41598-020-62544-4

Moreira, H., Pereira, S. I. A., Vega, A., Castro, P. M. L., and Marques, A. P. G. C. (2020). Synergistic effects of arbuscular mycorrhizal fungi and plant growth-promoting bacteria benefit maize growth under increasing soil salinity. J. Environ. Manag. 257:109982. doi: 10.1016/j.jenvman.2019.109982

Myouga, F., Hosoda, C., Umezawa, T., Iizumi, H., Kuromori, T., Motohashi, R., et al. (2008). A heterocomplex of iron superoxide dismutases defends chloroplast nucleoids against oxidative stress and is essential for chloroplast development in Arabidopsis. Plant Cell 20, 3148-3162. doi: 10.1105/tpc.108. 061341

Noy-Meyr, I. (1973). Desert ecosystems: environment and producers. Annu. Rev. Ecol. Evol. Syst. 4, 25-51. doi: 10.1146/annurev.es.04.110173.000325

Ozgur, R., Uzilday, B., Sekmen, A. H., and Turkan, I. (2013). Reactive oxygen species regulation and antioxidant defence in halophytes. Funct. Plant Biol. 40, 832-847. doi: 10.1071/FP12389

Ozgur, R., Uzilday, B., Sekmen, A. H., and Turkan, I. (2015). The effects of induced production of reactive oxygen species in organelles on endoplasmic reticulum stress and on the unfolded protein response in Arabidopsis. Ann. Bot. 116, 541-553. doi: 10.1093/aob/mcv072

Pasricha, S., MacRae, J. I., Chua, H. H., Chambers, J., Boyce, K. L., McConville, M. J., et al. (2017). Extensive metabolic remodeling differentiates nonpathogenic and pathogenic growth forms of the dimorphic pathogen Talaromyces marneffei. Front. Cell. Infect. Microbiol. 7:368. doi: 10.3389/fcimb. 2017.00368

Pellegrini, L., Rohfritsch, O., Fritig, B., and Legrand, M. (1994). Phenylalanine ammonia-lyase in tobacco (molecular cloning and gene expression during the hypersensitive reaction to tobacco mosaic virus and the response to a fungal elicitor). Plant Physiol. 106, 877-886. doi: 10.1104/pp.106.3.877

Pinhero, R. G., Rao, M. V., Paliyath, G., Murr, D. P., and Fletcher, R. A. (1997). Changes in activities of antioxidant enzymes and their relationship to genetic and paclobutrazol-induced chilling tolerance of maize seedlings. Plant Physiol. 114, 695-704. doi: 10.1104/pp.114.2.695

Pusztahelyi, T., Holb, I. J., and Pócsi, I. (2015). Secondary metabolites in fungusplant interactions. Front. Plant Sci. 6:573. doi: 10.3389/fpls.2015.00573

Radhakrishnan, R., Khan, A. L., and Lee, I.-J. (2013). Endophytic fungal pre-treatments of seeds alleviates salinity stress effects in soybean plants. J. Microbiol. 51, 850-857. doi: $10.1007 / \mathrm{s} 12275-013-3168-8$
Rodriguez, R., Marticorena, C., Alarcón, D., Baeza, C., Cavieres, L., Finot, V. L., et al. (2008). Catálogo de las plantas vasculares de Chile. Gayana Bot. 75, 1-430. doi: 10.4067/S0717-66432018000100001

Rodriguez, R. J., Henson, J., Van Volkenburgh, E., Hoy, M., Wright, L., Beckwith, F., et al. (2008). Stress tolerance in plants via habitat adapted symbiosis. ISME J. 2, 404-416. doi: 10.1038/ismej.2007.106

Rodriguez, R. J., Redman, R. S., and Henson, J. M. (2004). The role of fungal symbioses in the adaptation of plants to high stress environments. Mitig. Adapt. Strateg. Glob. Chang. 9, 261-272. doi: 10.1023/B:MITI.0000029922.31110.97

Rodriguez, R. J., White, J. F., Arnold, A. E., and Redman, R. S. (2009). Fungal endophytes: diversity and functional roles. New Phytol. 182, 314-330. doi: 10. 1111/j.1469-8137.2009.02773.x

Ruiz, K. B., Aloisi, I., Del Duca, S., Canelo, V., Torrigiani, P., Silva, H., et al. (2016). Salares versus coastal ecotypes of quinoa: salinity responses in Chilean landraces from contrasting habitats. Plant Physiol. Biochem. 101, 1-13. doi: 10.1016/j.plaphy.2016.01.010

Ruiz, K. B., Rapparini, F., Bertazza, G., Silva, H., Torrigiani, P., and Biondi, S. (2017). Comparing salt-induced responses at the transcript level in a salares and coastal-lowland landrace of quinoa (Chenopodium quinoa Willd). Environ. Exp. Bot. 139, 127-142. doi: 10.1016/j.envexpbot.2017.05.003

Sales, C. R. G., Ribeiro, R. V., Silveira, J. A. G., Machado, E. C., Martins, M. O., and Lagôa, A. M. M. A. (2013). Superoxide dismutase and ascorbate peroxidase improve the recovery of photosynthesis in sugarcane plants subjected to water deficit and low substrate temperature. Plant Physiol. Biochem. 73, 326-336. doi: 10.1016/j.plaphy.2013.10.012

Sauer, D. B., and Burroughs, R. (1986). Disinfection of seed surfaces with sodium hypochlorite. Phytopathology 76, 745-749.

Shah, S. S., Shi, L., Li, Z., Ren, G., Zhou, B., and Qin, P. (2020). Yield, agronomic and forage quality traits of different quinoa (Chenopodium quinoa Willd.) genotypes in Northeast China. Agronomy 10:1908. doi: 10.3390/agronomy10121908

Sharma, N., Gupta, N. K., Gupta, S., and Hasegawa, H. (2005). Effect of $\mathrm{NaCl}$ salinity on photosynthetic rate, transpiration rate, and oxidative stress tolerance in contrasting wheat genotypes. Photosynthetica 43, 609-613. doi: 10.1007/ s11099-005-0095-x

Singh, L. P., Gill, S. S., and Tuteja, N. (2011). Unraveling the role of fungal symbionts in plant abiotic stress tolerance. Plant Signal. Behav. 6, 175-191. doi: 10.4161/psb.6.2.14146

Singleton, V. L., Orthofer, R., and Lamuela-Raventos, R. M. (1999). Analysis of total phenols and other oxidation substrates and antioxidants by means of Folin-Ciocalteau reagent. Methods Enzymol. 299, 152-178. doi: 10.1016/S00766879(99)99017-1

Tannous, J., Barda, O., Luciano-Rosario, D., Prusky, D., Sionov, E., and Keller, N. P. (2020). New insight into pathogenicity and secondary metabolism of the plant pathogen Penicillium expansum through deletion of the epigenetic reader SntB. Front. Microbiol. 11:610. doi: $10.3389 /$ fmicb. 2020.00610

Uchida, A., Jagendorf, A. T., Hibino, T., Takabe, T., and Takabe, T. (2002). Effects of hydrogen peroxide and nitric oxide on both salt and heat stress tolerance in rice. Plant Sci. 163, 515-523. doi: 10.1016/S0168-9452(02)00159-0

Van Elsas, J. D., Chiurazzi, M., Mallon, C. A., Elhottova, D., Kristufek, V., and Salles, J. F. (2012). Microbial diversity determines the invasion of soil by a bacterial pathogen. Proc. Natl. Acad. Sci. 109, 1159-1164. doi: 10.1073/pnas. 1109326109

Vasquez, E. A., Glenn, E. P., Guntenspergen, G. R., Brown, J. J., and Nelson, S. G. (2006). Salt tolerance and osmotic adjustment of Spartina alterniflora (Poaceae) and the invasive $\mathrm{M}$ haplotype of Phragmites australis (Poaceae) along a salinity gradient. Am. J. Bot. 93, 1784-1790. doi: $10.3732 /$ ajb.93.12.1784

Vinale, F., Nicoletti, R., Lacatena, F., Marra, R., Sacco, A., Lombardi, N., et al. (2017). Secondary metabolites from the endophytic fungus Talaromyces pinophilus. Nat. Prod. Res. 31, 1778-1785. doi: 10.1080/14786419.2017.1290624

Visagie, C., Houbraken, J., Frisvad, J. C., Hong, S. B., Klaassen, C., Perrone, G., et al. (2014). Identification and nomenclature of the genus Penicillium. Stud. Mycol. 78, 343-371. doi: 10.1016/j.simyco.2014.09.001 
White, J. F., and Torres, M. S. (2010). Is plant endophyte-mediated defensive mutualism the result of oxidative stress protection? Physiol. Plant. 138, 440-446. doi: 10.1111/j.1399-3054.2009.01332.x

Yilmaz, N., López-Quintero, C. A., Vasco-Palacios, A. M., Frisvad, J. C., Theelen, B., Boekhout, T., et al. (2016). Four novel Talaromyces species isolated from leaf litter from Colombian Amazon rain forests. Mycol. Prog. 15, 1041-1056. doi: 10.1007/s11557-016-1227-3

Zarea, M., Hajinia, S., Karimi, N., Goltapeh, E. M., Rejali, F., and Varma, A. (2012). Effect of Piriformospora indica and Azospirillum strains from saline or nonsaline soil on mitigation of the effects of $\mathrm{NaCl}$. Soil Biol. Biochem. 45, 139-146. doi: 10.1016/j.soilbio.2011.11.006

Zhang, F., Wang, Y., Liu, C., Chen, F., Ge, H., Tian, F., et al. (2019). Trichoderma harzianum mitigates salt stress in cucumber via multiple responses. Ecotoxicol. Environ. Saf. 170, 436-445. doi: 10.1016/j.ecoenv.2018.11.084

Zhu, J. K. (2001). Plant salt tolerance. Trends Plant Sci. 6, 66-71. doi: 10.3389/fpls. 2016.01787
Conflict of Interest: The authors declare that the research was conducted in the absence of any commercial or financial relationships that could be construed as a potential conflict of interest.

Publisher's Note: All claims expressed in this article are solely those of the authors and do not necessarily represent those of their affiliated organizations, or those of the publisher, the editors and the reviewers. Any product that may be evaluated in this article, or claim that may be made by its manufacturer, is not guaranteed or endorsed by the publisher.

Copyright (๑) 2022 González-Teuber, Contreras, Zúñiga, Barrera and BascuñánGodoy. This is an open-access article distributed under the terms of the Creative Commons Attribution License (CC BY). The use, distribution or reproduction in other forums is permitted, provided the original author(s) and the copyright owner(s) are credited and that the original publication in this journal is cited, in accordance with accepted academic practice. No use, distribution or reproduction is permitted which does not comply with these terms. 